\title{
Development of a Simple Food Frequency Questionnaire to Estimate Intakes of Calcium and Other Nutrients for the Prevention and Management of Osteoporosis
}

\author{
Kazuhiro UENISHI ${ }^{1}$, Hiromi IsHIDA ${ }^{2}$ and Kazutoshi NAKAMURA ${ }^{3}$ \\ ${ }^{1}$ Laboratory of Physiological Nutrition, and ${ }^{2}$ Laboratory of Administrative Dietetics, Kagawa Nutrition \\ University, Saitama 350-0288, Japan \\ ${ }^{3}$ Department of Community Preventive Medicine, Niigata University Graduate School of Medical and Dental \\ Sciences, Niigata 951-8510, Japan
}

(Received May 1, 2007)

\begin{abstract}
Summary There have been no simple methods to estimate dietary nutrient intakes for the prevention and management of osteoporosis. The aim of this study was to develop and validate a new, simple food frequency questionnaire (FFQ) for dietary intake of calcium and other nutrients relevant to the bone health of adult Japanese women. We developed a 28item FFQ. To validate this, 208 and 72 adult women aged between 18 and 69 y were recruited for testing reliability and reproducibility, respectively. In the 208 women, moderate-to-high Spearman's correlation coefficients between our FFQ and the conventional diet record method were found in intakes of calcium $(r=0.668)$, sodium chloride $(\mathrm{NaCl})$ $(r=0.475)$, vitamin A $(r=0.501)$, vitamin D $(r=0.413)$, vitamin $\mathrm{K}(r=0.649)$, and energy $(r=0.471)$. In the 72 women, coefficients of variance of the four repeated measurements of intakes throughout a year were $14.1 \%$ for calcium, $7.3 \%$ for $\mathrm{NaCl}, 21.2 \%$ for vitamin A, $13.6 \%$ for vitamin D, $36.8 \%$ for vitamin K, and $9.6 \%$ for energy. In conclusion, the FFQ we developed is a useful tool to evaluate the intake of dietary calcium of adult Japanese women. Although it can also measure intakes of dietary vitamin A, vitamin D, vitamin $\mathrm{K}, \mathrm{NaCl}$, and energy, further improvement is needed to measure intakes of these nutrients and energy.

Key Words dietary calcium, food frequency questionnaire, Japan, osteoporosis, validation studies
\end{abstract}

Osteoporosis is characterized by generalized skeletal fragility, and fractures due to osteoporosis have rapidly increased in Japan (1). In this situation, prevention and management of this disease is becoming more enhanced in clinical medicine and public health.

Calcium is an essential nutrient for bone health because its insufficient intake could adversely affect age-related bone loss and attainment of peak bone mass. Likewise, other dietary nutrients, such as vitamin $\mathrm{D}$ and vitamin $\mathrm{K}$ are known to have an important role for human bone metabolism. Therefore, assessment of intakes of these nutrients from diet is meaningful from the viewpoint of the prevention and management of osteoporosis.

There are several methods to estimate calcium intake. Among them, a food frequency questionnaire (FFQ) is simpler than other methods, such as a diet record or a duplicate portion sampling method, which is heavy a burden for a person to complete. Furthermore, the FFQ has another advantage, enhancing longterm intake of nutrients better than the 24-h recall method (2). For these reasons, the FFQ is considered to be one of the best tools to estimate calcium intake for the prevention and management of osteoporosis. Some

E-mail: uenishi@eiyo.ac.jp
FFQs have been developed to estimate intakes of a wide range of nutrients, including calcium, for Japanese adults (3, 4). However, these FFQs currently used in Japan have many questions and they take a long time to finish, and thus an even shorter FFQ is needed for use in epidemiological and clinical settings. In European and North American countries, there are several simple FFQs specifically developed for estimating calcium intake (5-8); however, there have been few such FFQs validated for Japanese adults.

Recently, some other nutrients, such as iron, sodium chloride $(\mathrm{NaCl})$, vitamin $\mathrm{A}$, vitamin $\mathrm{D}$, and vitamin $\mathrm{K}$, have been suggested to be associated with bone mass (9), and therefore, monitoring intakes of these nutrients as well as calciums is becoming of importance. Nonetheless, there have been no simple methods to estimate dietary intakes of these nutrients. The aim of this study was to develop and validate a simple FFQ for the prevention and management of osteoporosis (FFQPOP) to estimate dietary intake of calcium and other relevant nutrients of adult Japanese women.

\section{METHODS}

Procedure for the development of an FFQ. The FFQPOP we developed was based on the semi-quantified food frequency questionnaire developed by the Drafting Com- 
Table 1. Mean values of nutrients and energy measured by the FFQPOP ${ }^{1}$ and the diet record method in 208 subjects, and Pearson's product moment correlation coefficients of values between the two methods.

\begin{tabular}{|c|c|c|c|c|c|}
\hline \multirow{2}{*}{ Nutrients or energy } & \multicolumn{2}{|c|}{ Mean values (SD) } & \multirow{2}{*}{$\begin{array}{l}\text { Relative difference } \\
\text { to diet record (\%) }\end{array}$} & \multicolumn{2}{|c|}{ Correlation coefficients ${ }^{2}$} \\
\hline & FFQPOP & Diet record & & Pearson's & Spearman's \\
\hline Calcium (mg/d) & $476(155)$ & $522(216)$ & -9 & 0.676 & 0.668 \\
\hline $\operatorname{Iron}(\mathrm{mg} / \mathrm{d})$ & $6.9(2.0)$ & $6.6(3.7)$ & 5 & 0.500 & 0.394 \\
\hline $\mathrm{NaCl}(\mathrm{g} / \mathrm{d})$ & $9.9(1.9)$ & $7.7(2.2)$ & 29 & 0.467 & 0.475 \\
\hline Vitamin A ( $\mu \mathrm{g} R E / d)$ & $797(421)$ & $727(641)$ & 10 & 0.709 & 0.501 \\
\hline Vitamin $\mathrm{D}(\mu \mathrm{g} / \mathrm{d})$ & $9.2(2.3)$ & $6.5(4.7)$ & 42 & 0.403 & 0.413 \\
\hline $\operatorname{Vitamin} \mathrm{K}(\mu \mathrm{g} / \mathrm{d})$ & $157(108)$ & $232(161)$ & -32 & 0.669 & 0.649 \\
\hline Energy (kcal/d) & $1,621(294)$ & $1,625(309)$ & 0 & 0.415 & 0.471 \\
\hline Protein $(\mathrm{g} / \mathrm{d})$ & $70.1(18.1)$ & $58.9(14.9)$ & 19 & 0.319 & 0.339 \\
\hline Lipid (g/d) & $62.2(15.7)$ & $52.0(15.5)$ & 20 & 0.307 & 0.345 \\
\hline Carbohydrate (g/d) & $197(38)$ & $225(46)$ & -12 & 0.394 & 0.299 \\
\hline
\end{tabular}

${ }^{1}$ Food frequency questionnaire for the prevention and management of osteoporosis we developed.

${ }^{2} p<0.001$ for all.

mittee of the Ministry of Health and Welfare for Health Index (10). We selected 19 food items, which are major sources of calcium among Japanese foods, from the questionnaire. In addition, we adopted 9 items, such as nuts and liver, whose calcium content is low but which are major sources of other nutrients related to bone health, such as vitamin $\mathrm{A}$, vitamin $\mathrm{D}$, vitamin $\mathrm{K}$, and energy. In total, the FFQPOP included 28 food items. The FFQPOP is shown in Appendix 1, in which each item includes 3 to 5 grades of frequency of food intake. Subjects were asked to select a grade of frequency in the last $1 \mathrm{mo}$. Intakes of the nutrients and energy were estimated by a grade of frequency multiplied by the relevant coefficients determined on the basis of the Standard Tables of Food Composition in Japan (11).

Subjects for testing reliability and reproducibility of the FFOPOP. The FFQPOP was validated by setting three populations: 1) 190 female college students aged between 18 and $22 \mathrm{y}$, and 18 adult females aged between 46 and $69 \mathrm{y}$ to compare mean values of nutrients and energy between the FFQPOP and a conventional method of 3-d diet record with weighing used in the National Health and Nutrition Survey in Japan (12), 2) 30 female college students aged between 19 and $22 \mathrm{y}$, and 42 adult females aged between 46 and $61 \mathrm{y}$ to evaluate reproducibility by measuring nutrients and energy with the FFQPOP four times a year (every $3 \mathrm{mo}$ ), and 3) 190 female college students aged between 18 and $22 \mathrm{y}$, and 234 adult females aged between 50 and $69 \mathrm{y}$ to compare mean values of nutrients and energy of the FFQPOP with the results of the National Health and Nutrition Survey in Japan (2004) (12).

Statistical methods. Relative differences in values of nutritional intake obtained from the FFQPOP to the dietary record method were calculated. Spearman's correlation coefficients were mainly used to test a correlation between FFQPOP and the diet record method, although both Pearson's and Spearman's correlation coefficients are presented. Coefficients of variance (CVs) were calculated to show a variation of repeated measurements for nutritional intakes.

\section{RESULTS}

\section{Comparison with the diet record method}

Average intakes of nutrients and energy measured by the FFQPOP and the diet record method, and correlation coefficients between the two methods are shown in Table 1. High correlation coefficients of near 0.7 were found in intakes of calcium and vitamin $\mathrm{K}$. The relative difference between the FFQPOP and the diet record method was small in calcium $(-9 \%)$, but the relative difference in vitamin $\mathrm{K}$ was larger $(-32 \%)$ than that of calcium. Moderate correlation coefficients around 0.5 were found in intakes of $\mathrm{NaCl}$, vitamin $\mathrm{A}$, vitamin $\mathrm{D}$ and energy. Correlation coefficients for the other nutrients were low.

Reproducibility

Average intakes of nutrients and energy measured four times by the FFQPOP are shown in Table 2. CVs of the four repeated measurements were around $10 \%$ except for vitamin A (21.2\%) and vitamin K (36.8\%). Comparison with results of the National Health and Nutrition Survey in Japan

Average intake of nutrients and energy measured by the FFQPOP and the results of the National Health and Nutrition Survey in Japan (2004) (12) in the 18-22-yage group and the 50s-and-60s group are shown in Table 3. Relative differences to the National Health and Nutrition Survey tended to be negative except in calcium and vitamin $\mathrm{K}$ in the 18-22-y-age group, whereas they tended to be positive except in energy. A large difference was observed in vitamin D intake in the 50sand-60s group.

\section{DISCUSSION}

This study demonstrated a high correlation $(r=0.67)$ of calcium intakes between the FFQPOP and the diet record method, which implies that the FFQPOP is valid on the individual level. Furthermore, levels of calcium 
Table 2. Mean values of nutrients and energy measured repeatedly by the FFQPOP ${ }^{1}$ and their coefficients of variance (CVs) in 72 subjects.

\begin{tabular}{|c|c|c|c|c|c|c|}
\hline \multirow{3}{*}{ Nutrients or energy } & \multicolumn{4}{|c|}{ Mean value (SD) } & \multirow{3}{*}{$\begin{array}{l}\text { Mean of the } 4 \\
\text { measurements }\end{array}$} & \multirow{3}{*}{$\mathrm{CV}(\%)$} \\
\hline & \multicolumn{4}{|c|}{ Measurements } & & \\
\hline & $1 \mathrm{st}$ & $2 \mathrm{nd}$ & $3 \mathrm{rd}$ & 4 th & & \\
\hline Calcium (mg/d) & $562(159)$ & $485(157)$ & $523(148)$ & $495(151)$ & 517 & 14.1 \\
\hline $\operatorname{Iron}(\mathrm{mg} / \mathrm{d})$ & $8.5(2.0)$ & $7.4(1.9)$ & $7.7(1.9)$ & $7.3(2.2)$ & 7.7 & 15.9 \\
\hline $\mathrm{NaCl}(\mathrm{g} / \mathrm{d})$ & $10.3(1.9)$ & $9.8(1.4)$ & $10.2(1.9)$ & $9.8(1.6)$ & 10.0 & 7.3 \\
\hline Vitamin A ( $\mu \mathrm{g}$ RE/d) & $977(430)$ & $934(388)$ & $958(552)$ & $910(478)$ & 945 & 21.2 \\
\hline Vitamin $\mathrm{D}(\mu \mathrm{g} / \mathrm{d})$ & $10.6(2.5)$ & $10.0(2.3)$ & $10.2(2.2)$ & $10.5(2.4)$ & 10.3 & 13.6 \\
\hline Vitamin $\mathrm{K}(\mu \mathrm{g} / \mathrm{d})$ & $123(32)$ & $115(35)$ & $213(112)$ & $148(81)$ & 150 & 36.8 \\
\hline Energy $(\mathrm{kcal} / \mathrm{d})$ & $1,642(276)$ & $1,594(247)$ & $1,623(233)$ & $1,602(284)$ & 1,615 & 9.6 \\
\hline Protein $(\mathrm{g} / \mathrm{d})$ & $72.7(15.5)$ & $71.9(12.6)$ & $71.1(11.6)$ & $69.9(14.3)$ & 71.4 & 12.0 \\
\hline Lipid $(g / d)$ & $62.6(14.5)$ & $59.1(11.1)$ & $62.5(11.9)$ & $61.0(12.0)$ & 61.3 & 12.4 \\
\hline Carbohydrate (g/d) & $202(39)$ & $198(40)$ & $199(40)$ & $198(49)$ & 199 & 11.0 \\
\hline
\end{tabular}

${ }^{1}$ Food frequency questionnaire for the prevention and management of osteoporosis we developed.

Table 3. Mean values of major nutrients and energy measured by the FFQPOP ${ }^{1}$ in 424 subjects and the results of the National Health and Nutrition Survey in Japan (2004) (12) by age groups in females.

\begin{tabular}{|c|c|c|c|c|c|c|}
\hline \multirow{3}{*}{ Nutrients or energy } & \multicolumn{3}{|c|}{ 18-22-y-age group } & \multicolumn{3}{|c|}{ 50s-and-60s group } \\
\hline & \multicolumn{2}{|c|}{ Mean (SD) } & \multirow{2}{*}{$\begin{array}{l}\text { Relative } \\
\text { difference } \\
\text { to NHNS }(\%)\end{array}$} & \multicolumn{2}{|c|}{ Mean (SD) } & \multirow{2}{*}{$\begin{array}{l}\text { Relative } \\
\text { difference } \\
\text { to NHNS (\%) }\end{array}$} \\
\hline & FFQPOP & $\mathrm{NHNS}^{2}$ & & FFQPOP & $\mathrm{NHNS}^{2}$ & \\
\hline Calcium (mg/d) & $503(200)$ & $429(206)$ & 17 & $585(166)$ & $568(286)$ & 3 \\
\hline Iron $(\mathrm{mg} / \mathrm{d})$ & $6.2(1.9)$ & $6.8(2.6)$ & -9 & $9.4(2.5)$ & $8.7(3.7)$ & 8 \\
\hline $\mathrm{NaCl}(\mathrm{g} / \mathrm{d})$ & $7.5(2.0)$ & $9.4(3.5)$ & -20 & $10.9(1.9)$ & $11.2(4.4)$ & -3 \\
\hline Vitamin A ( $\mu \mathrm{g} \mathrm{RE} / \mathrm{d})$ & $634(301)$ & $740(650)$ & -13 & $1164(701)$ & $974(778)$ & 20 \\
\hline $\operatorname{Vitamin} \mathrm{D}(\mu \mathrm{g} / \mathrm{d})$ & $6.0(4.5)$ & $6.0(8.4)$ & 0 & $12.7(2.5)$ & $9.1(9.5)$ & 40 \\
\hline Vitamin $\mathrm{K}(\mu \mathrm{g} / \mathrm{d})$ & $224(155)$ & $212(179)$ & 6 & $316(149)$ & $290(237)$ & 9 \\
\hline Energy $(\mathrm{kcal} / \mathrm{d})$ & $1,602(303)$ & $1,670(458)$ & -4 & $1,702(292)$ & $1,790(469)$ & -5 \\
\hline Protein $(\mathrm{g} / \mathrm{d})$ & $57.0(13.3)$ & $62.7(19.0)$ & -9 & $76.4(17.2)$ & $70.5(22.6)$ & 8 \\
\hline $\operatorname{Lipid}(\mathrm{g} / \mathrm{d})$ & $51.4(15.6)$ & $54.0(22.6)$ & -5 & $60.6(13.1)$ & $49.3(21.0)$ & 23 \\
\hline Carbohydrate (g/d) & $223(45)$ & $225(68)$ & -1 & $217(42)$ & $258(73)$ & -16 \\
\hline
\end{tabular}

${ }^{1}$ Food frequency questionnaire for the prevention and management of osteoporosis we developed.

${ }^{2}$ National Health and Nutrition Survey in Japan (2004) (12).

intake estimated by the FFQPOP in this study are comparable to the results of the National Health and Nutrition Survey, suggesting the FFOPOP is valid on the population level. Because of these reasons, the FFQPOP is primarily useful in the assessment of calcium intake for epidemiologic studies and health education in clinical settings.

In general, an acceptable correlation coefficient between an FFQ and the conventional diet record have been considered between 0.5 and 0.7 (2). The correlation coefficient of calcium intake between the FFQPOP and the diet record method was as high as 0.67. This value is better than those (0.52 and 0.53) of general FFQs for Japanese people previously reported $(3,4)$. Some FFQs have specifically been developed for assessing dietary calcium intake. There have been two FFQs assessing dietary calcium intake for Asians. Sato et al. (13) developed a simple FFQ for estimating calcium intake for Japanese with a correlation coefficient of 0.51 (vs. 1-d food record), and Chee et al. (14) developed such an FFQ for Malaysian women with a correlation coefficient of 0.56 (vs. 3-d food record). Conversely, FFQs assessing calcium intake in the Western adult diet have yielded a correlation coefficient of $0.64-0.90$ between the FFQ and gold standard methods (5-8). Their values are higher than those of calcium FFQs previously developed for Asians $(13,14)$. Calcium FFQs for North Americans and Europeans generally have good correlation coefficients probably because the amount of calcium intake is higher on average and ranges wider than those for Asians, and because their sources of calcium are simple, depending largely on milk and milk products. Sources of calcium for the Japanese diet are more varied than for the Western diet, and are from dried baby fish, colored vegetables, seaweed, and beans (and bean products) in addition to milk and milk prod- 
ucts (12). Despite these facts, the FFQPOP had a similar correlation level to those for Western FFQs. The CV for calcium intake by the FFQPOP was 14\%, and its relative difference to National Health and Nutrition Survey was $3-17 \%$, which are acceptable levels. Therefore, the FFQPOP is satisfactorily valid and reproducible to estimate calcium intake.

There have been no simple FFQs to estimate nutrients other than calcium related to bone health. This study first demonstrated that intakes of vitamin $\mathrm{K}$ can be measured by the FFQPOP with high correlation to the diet record method. It should be cautioned, however, that the difference in vitamin $\mathrm{K}$ intake between the FFQPOP and the diet record method is relatively large, and that reproducibility for intakes of vitamin $\mathrm{K}$ is poorer than for other nutrients.

Moderate correlation coefficients between the FFQPOP and the diet record method were found for $\mathrm{NaCl}$, vitamin A, vitamin D, and energy. Among them, the absolute vitamin D intake of the FFQPOP showed a relatively large difference from that of the diet record and National Health and Nutrition Survey (older women only). This should be taken into account, although the primary purpose of an FFQ is not necessarily to obtain the accurate absolute value of a nutritional intake, but to obtain its relative levels in a target population.

There are some limitations in this study. First, the 3-d diet record may not accurately estimate some nutrient intakes such as vitamins although it is often used as a gold standard in many validation studies. Basiotis et al. (15) suggested that a greater number of days of vitamin A intake is required than for energy and minerals. This may be the case for other vitamins, such as vitamin D and vitamin K. Ideally, vitamins in this FFQ should further be evaluated with a more reliable gold standard method. Second, some vitamins, such as vitamin $\mathrm{K}$ and vitamin A were relatively poorly reproducible. Their large CV values may probably be due to a seasonal variation in their intakes. Although the present reproducibility was potentially affected by the seasonal variations as a limitation, CVs of other nutrients and energy were relatively small. Finally, general nutrients, such as protein, could affect bone health, but their dietary intakes were not estimated with sufficient reliability. These limitations should be addressed by modifying this FFQPOP in future studies.

The FFQPOP we developed has several strengths. First, the FFQPOP is simple and convenient. It consists of only 28 questions, and it takes about $10 \mathrm{~min}$ to complete. Therefore, the FFQPOP can be used not only in an epidemiologic study, but also in clinical settings. Second, dietary calcium intake can be assessed with excellent validity and reproducibility. Third, multiple nutrients related to bone health and osteoporosis can be evaluated. In conclusion, the FFQPOP is a useful tool to evaluate the intake of dietary calcium in adult Japanese women for prevention and management of osteoporosis. Although it can also measure intakes of dietary vitamin $\mathrm{A}$, vitamin $\mathrm{D}$, vitamin $\mathrm{K}, \mathrm{NaCl}$, and energy, fur- ther improvement is needed to measure intakes of these nutrients and energy.

\section{Acknowledgments}

The authors thank Dr. Masataka Shiraki (Research Institute and Practice for Involutional Diseases, Nagano, Japan) and Megumi Kubota (Okayama Prefectural University, Okayama, Japan) for their help.

\section{REFERENCES}

1) Morita Y, Endo N, Iga T, Tokunaga K, Ohkawa Y. 2002. The incidence of cervical and trochanteric fractures of the proximal femur in 1999 in Niigata Prefecture, Japan. J Bone Miner Metab 20: 311-318.

2) Willett W. 1990. Nutritional Epidemiology, p 69-126. Oxford University Press, New York.

3) Egami I, Wakai K, Kato K, Lin Y, Kawamura T, Tamakoshi A, Aoki R, Kojima M, Nakayama T, Wada M, Ohno Y. 1999. A simple food frequency questionnaire for Japanese diet-Part II. Reproducibility and validity for nutrient intakes. J Epidemiol 9: 227-234.

4) Tsugane S, Kobayashi M, Sasaki S. 2003. Validity of the self-administered food frequency questionnaire used in the 5-year follow-up survey of the JPHC Study Cohort I: comparison with dietary records for main nutrients. $J$ Epidemiol 13 (1 Suppl): S51-56.

5) Cummings SR, Block G, McHenry K, Baron RB. 1987. Evaluation of two food frequency methods of measuring dietary calcium intake. Am J Epidemiol 126: 796-802.

6) Musgrave KO, Giambalvo L, Leclerc HL, Cook RA, Rosen CJ. 1989. Validation of a quantitative food frequency questionnaire for rapid assessment of dietary calcium intake. J Am Diet Assoc 89: 1484-1488.

7) Montomoli M, Gonnelli S, Giacchi M, Mattei R, Cuda C, Rossi S, Gennari C. 2002. Validation of a food frequency questionnaire for nutritional calcium intake assessment in Italian women. Eur J Clin Nutr 56: 21-30.

8) Magkos F, Manios Y, Babaroutsi E, Sidossis LS. 2006. Development and validation of a food frequency questionnaire for assessing dietary calcium intake in the general population. Osteoporos Int 17: 304-312.

9) Ilich JZ, Kerstetter JE. 2000. Nutrition in bone health revisited: a story beyond calcium. J Am Coll Nutr 19: $715-737$.

10) Morimoto A, Takase S, Hada K, Hosoya N. 1977. Evaluation of a "convenient method" for the estimation of dietary food consumptions. Jpn J Nutr 35: 235-245 (in Japanese).

11) Resources Council, Science and Technology Agency, Japan. 2000. Standard Tables of Food Composition in Japan, 5th ed. Printing Bureau, Ministry of Finance, Tokyo (in Japanese).

12) Kenko-eiyo-joho-kenkyukai. 2004. The National Health and Nutrition Survey in Japan, 2002. Daiichi Shuppan Publisher, Tokyo.

13) Sato Y, Tamaki J, Kitayama F, Kusaka Y, Kodera Y, Koutani A, Iki M. 2005. Development of a food-frequency questionnaire to measure the dietary calcium intake of adult Japanese women. Tohoku J Exp Med 207: 217222.

14) Chee WS, Suriah AR, Zaitun Y, Chan SP, Yap SL, Chan YM. 2002. Dietary calcium intake in postmenopausal Malaysian women: comparison between the food frequency questionnaire and three-day food records. Asia 
Pac J Clin Nutr 11: 142-146.

15) Basiotis PP, Welsh SO, Cronin FJ, Kelsay JL, Mertz W. 1987. Number of days of food intake records required to estimate individual and group nutrient intakes with defined confidence. J Nutr 117: 1638-1641.

Appendix 1. The food frequency questionnaire for the prevention and management of osteoporosis (FFQPOP).

\begin{tabular}{|c|c|c|c|}
\hline & Food items & Standard portion size & Frequency or amount \\
\hline 1 & Milk & $200 \mathrm{~mL}$ & none, sometimes, $1 / \mathrm{d},(\quad) \mathrm{mL} / \mathrm{d}$ \\
\hline \multirow[t]{4}{*}{2} & Milk products & & \\
\hline & Yogurt & $100 \mathrm{~g}$ & rarely, $1-3 / \mathrm{wk}, 4-6 / \mathrm{wk}, 1 / \mathrm{d},(\quad) / \mathrm{d}$ \\
\hline & Cheese & $20 \mathrm{~g}$ & rarely, $1-3 / w k, 4-6 / w k, 1 / d,(\quad) / d$ \\
\hline & Skim milk & $10 \mathrm{~g}$ & rarely, $1-3 / w k, 4-6 / w k, 1 / d,(\quad) / d$ \\
\hline 3 & Egg & one & none, sometimes, $1 / \mathrm{d},(\quad) / \mathrm{d}$ \\
\hline 4 & Fish & $80 \mathrm{~g}$ & none, sometimes, $1 / \mathrm{d},(\quad) / \mathrm{d}$ \\
\hline 5 & Shellfish & one dish & rarely, $1-2 / \mathrm{mo}, 1-3 / \mathrm{wk}, \geq 4 / \mathrm{wk}$ \\
\hline 6 & Squid, octopus, prawn & one dish & rarely, $1-2 / \mathrm{mo}, 1-3 / \mathrm{wk}, \geq 4 / \mathrm{wk}$ \\
\hline 7 & $\begin{array}{l}\text { Dried baby fish } \\
\text { (Shirasuboshi, Jako, etc.) }\end{array}$ & $10 \mathrm{~g}$ & rarely, $1-2 / \mathrm{mo}, 1-3 / \mathrm{wk}, \geq 4 / \mathrm{wk}$ \\
\hline 8 & Fish eggs (Ikura, Tarako, etc.) & one dish & rarely, $1-2 / \mathrm{mo}, 1-3 / \mathrm{wk}, \geq 4 / \mathrm{wk}$ \\
\hline 9 & Meat or meat products & $100 \mathrm{~g}$ & none, sometimes, $1 / \mathrm{d},(\quad) / \mathrm{d}$ \\
\hline 10 & Liver & $50 \mathrm{~g}$ & rarely, $1-2 / \mathrm{mo}, 1-3 / \mathrm{wk}, \geq 4 / \mathrm{wk}$ \\
\hline 11 & Beans or bean products & $1 / 4$ Tofu & rarely, small size/d, medium size/d, large size/d \\
\hline 12 & Natto (fermented soy bean) & 1 pack & rarely, $1-3 / w k, 4-6 / w k, 1 / d,(\quad) / d$ \\
\hline 13 & Potatoes & $50 \mathrm{~g}$ & rarely, small size/d, medium size/d, large size/d \\
\hline 14 & Vegetables (green and yellow) & $70 \mathrm{~g}$ & rarely, small size/d, medium size/d, large size/d \\
\hline 15 & Vegetables (other) & $70 \mathrm{~g}$ & rarely, small size/d, medium size/d, large size/d \\
\hline 16 & Seaweed & one dish & rarely, $1-3 / w k, 1 / \mathrm{d}, \geq 2 / \mathrm{d}$ \\
\hline 17 & Nuts & $20 \mathrm{~g}$ & rarely, $1-3 / w k, 4-6 / w k, 1 / d$ \\
\hline 18 & Fruit (apple, pear, orange, etc.) & one & rarely, a half/d, 1/d, ( )/d \\
\hline \multirow[t]{4}{*}{19} & Staple food & & \\
\hline & Rice & one bowl & none, $($ )/d \\
\hline & Bread & one slice & none, $($ )/d \\
\hline & Noodles & one bowl & none, $(\quad) / d$ \\
\hline 20 & Butter, margarine, jam with bread & $10 \mathrm{~g}$ & none, spread thin, spread thick \\
\hline 21 & Miso soup & one bowl & rarely, $1-3 / w k, 4-6 / w k, 1 / d,(\quad) / d$ \\
\hline 22 & Coffee or tea & $120 \mathrm{~mL}$ & none, sometimes, $1 / \mathrm{d},(\quad) / \mathrm{d}$ \\
\hline 23 & Sugar with coffee or tea & $4 \mathrm{~g}$ & none, one portion, ( ) portion \\
\hline 24 & Sweet beverages & $250 \mathrm{~mL}$ & none, sometimes, $(\quad) \mathrm{mL} / \mathrm{d}$ \\
\hline 25 & Sweets & one & rarely, sometimes, $1 / \mathrm{d}$ \\
\hline 26 & Greasy dish & - & rarely, sometimes, $1 / \mathrm{d},(\quad) / \mathrm{d}$ \\
\hline 27 & Pickles & one dish & rarely, sometimes, $1 / \mathrm{d}, \geq 2 / \mathrm{d}$ \\
\hline 28 & Alcohol & one unit & rarely, $1-3 / w k, 4-6 / w k, 1 / d,(\quad) / d$ \\
\hline
\end{tabular}

